\title{
INVARIANCE PRINCIPLES UNDER A TWO-PART MIXING ASSUMPTION
}

\author{
Richard C. BRADLEY* \\ Department of Mathematics, Indiana University, Bloomington, IN 47405, and \\ Center for Stochastic Processes, Department of Statistics, University of North Carolina, \\ Phillips Hall 039A, Chapel Hill, NC 27514, USA \\ Magda PELIGRAD** \\ Department of Mathematics, University of Cincinnati, Cincinnati, OH 45221, USA
}

Received 25 September 1985

\begin{abstract}
Weak and strong invariance principles are established for strictly stationary sequences satisfying a mixing assumption which has two "parts", one based on the strong mixing condition with a polynomial mixing rate and the other based on the $\rho$-mixing condition.
\end{abstract}

AMS 1980 Subject Classifications: Primary 60G10; Secondary $60 \mathrm{~F} 05$.

strictly stationary sequence $*$ strong mixing conditions $*$ weak and strong invariance principles

\section{Introduction}

Suppose $\left\{X_{k}, k \in \mathbb{Z}\right\}$ is a strictly stationary sequence of (real valued) random variables on a probability space $(\Omega, \mathscr{F}, P)$. For $-\infty \leqslant J \leqslant L \leqslant \infty$ define $\mathscr{F}_{J}^{L}:=$ $\sigma\left(X_{k}, J \leqslant k \leqslant L\right)$. For each $n \geqslant 1$ define

$$
\begin{aligned}
& \alpha(n):=\sup |P(A \cap B)-P(A) P(B)|, \quad A \in \mathscr{F}_{-\infty}^{0}, \quad B \in \mathscr{F}_{n}^{\infty} ; \\
& \rho(n):=\sup |\operatorname{Corr}(f, g)|, \quad f \in \mathscr{L}_{2}\left(\mathscr{F}_{-\infty}^{0}\right), \quad g \in \mathscr{L}_{3}\left(\mathscr{F}_{n}^{\infty}\right) ; \\
& \varphi(n):=\sup |P(B \mid A)-P(B)|, \quad A \in \mathscr{F}_{-\infty}^{0}, B \in \mathscr{F}_{n}^{\infty}, P(A)>0 .
\end{aligned}
$$

The sequence is said to be "strongly mixing" if $\alpha(n) \rightarrow 0$ as $n \rightarrow \infty$, " $\rho$-mixing" if $\rho(n) \rightarrow 0$ as $n \rightarrow \infty$, and " $\varphi$-mixing" if $\varphi(n) \rightarrow 0$ as $n \rightarrow \infty$. It is well known that $\rho$-mixing implies strong mixing and (see Ibragimov and Linnik [18, Theorem 17.2.3]) $\varphi$-mixing implies $\rho$-mixing.

Mixing types of dependence lead to many useful limit theorems with broad applicability in statistical mechanics (see e.g. Denker and Philipp [12]) or statistics in general. In order to establish the central limit theorem for strongly mixing

* Partially supported by NSF grant DMS 84-01021 and AFOSR grant \#F49620 82 C 0009.

** Partially supported by NSF grant DMS 85-03016. 
sequences, the most instrumental way is to find a good estimate for the rate of convergence of $\alpha(n)$ to zero. In some situations it is difficult to compute this rate, and one has to look for an alternative approach such as verifying the $\rho$-mixing or even $\varphi$-mixing condition (perhaps without concern for mixing rate). When this approach also fails, there is another possibility: to show that there exists a sequence $\left\{D_{n}\right\}$ of "large nice events", with $P\left(D_{n}\right) \rightarrow 1$ as $n \rightarrow \infty$, such that for large $n$ the sequence $\left\{X_{k}\right\}$ restricted to $D_{n}$ has a small value for $\rho(n)$ or $\varphi(n)$, converging to zero as $n \rightarrow \infty$. One might refer to such a condition as a "restricted $\rho$-mixing" or "restricted $\varphi$-mixing" condition. This is a little vague, but as a specific example the well known "absolute regularity" (weak Bernoulli) condition can be formulated as a "restricted $\varphi$-mixing" condition; see Shields [24,p. 89]. This condition was studied in many papers on limit theory for dependent random variables; see e.g. Volkonskii and Rozanov [25], Gastwirth and Rubin [14], Yoshihara [26], Berbee [1], and Dehling and Philipp [11].

The purpose of this paper is to study limit theory under "restricted $\rho$-mixing" conditions. Now the $\rho$-mixing condition itself can be formulated in terms of pairs of events, as follows (see [6], [7, Thenrem 1.1(ii)], or [8]):

$$
\begin{aligned}
\rho_{0}(n) & :=\left[\sup \frac{|P(A \cap B)-P(A) P(B)|}{[P(A) P(B)]^{1 / 2}}, A \in \mathscr{F}_{-\infty}^{0}, B \in \mathscr{F}_{n}^{\infty}, P(A) P(B)>0\right] \\
& \rightarrow 0 \text { as } n \rightarrow \infty .
\end{aligned}
$$

One can formulate various "restricted $\rho$-mixing" conditions depending on whether one uses pairs of events or pairs of $\mathscr{L}_{2}$-functions, on whether (for each $n$ ) one "conditions" on the "large nice event" $D_{n}$ or uses some other way of analyzing the sequence $\left\{X_{k}\right\}$ restricted to $D_{n}$, and also on whether extra assumptions are imposed on $D_{n}$ (e.g. $D_{n} \in \mathscr{F}_{-\infty}^{0}$ ). Among these conditions, the following seems to be one of the lcast restrictive: There exist sequences $\left\{a_{n}\right\}$ and $\left\{\lambda_{n}\right\}$ of nonnegative real numbers, with $a_{n} \rightarrow 0$ and $\lambda_{n} \rightarrow 0$ as $n \rightarrow \infty$, such that

$$
\forall n \geqslant 1 \exists D_{n} \in \mathscr{F} \text { such that }
$$

(i) $P\left(D_{n}\right) \geqslant 1-a_{n}$, and

(ii) $\forall A \in \mathscr{F}_{-\infty}^{0} \forall B \in \mathscr{F}_{n}^{\infty}$

$$
\begin{aligned}
& \left|P\left(A \cap B \cap D_{n}\right)-P\left(A \cap D_{n}\right) P\left(B \cap D_{n}\right)\right| \\
& \quad \leqslant \lambda_{n} \cdot\left[P\left(A \cap D_{n}\right) P\left(B \cap D_{n}\right)\right]^{1 / 2} .
\end{aligned}
$$

A careful but simple calculation will show that (1.1) implies

$$
\begin{aligned}
& \forall n \geqslant 1 \forall A \in \mathscr{F}_{-\infty}^{0} \forall B \in \mathscr{F}_{n}^{\infty} \\
& |P(A \cap B)-P(A) P(B)| \leqslant a_{n}+\lambda_{n}[P(A) P(B)]^{1 / 2}
\end{aligned}
$$

for the same sequences $\left\{a_{n}\right\}$ and $\left\{\lambda_{n}\right\}$. Indeed, (1.2) seems to be implied by, as well as being similar to but also simpler than, any reasonable version of a "restricted $\rho$-mixing" condition as described above. Accordingly, our purpose will be well 
served by simply studying limit theory under condition (1.2); this is the condition that will be used in all of our results.

The conditions (1.1) and (1.2) (with $a_{n} \rightarrow 0$ and $\lambda_{n} \rightarrow 0$ ) can be described as "two-part" mixing conditions, and each implies strong mixing with rate $\alpha(n) \leqslant$ $a_{n}+\lambda_{n}$. It is well known that strong mixing does not imply absolute regularity. We conjecture that condition (1.1) (that is, the assumption that there exists $a_{n} \rightarrow 0$ and $\lambda_{n} \rightarrow 0$ such that (1.1) holds) likewise describes a proper subclass of the strongly mixing stationary sequences.

On the other hand strong mixing implies (1.2) (with $a_{n}:=\alpha(n)$ and $\lambda_{n}:=0$ ). The difference between (1.2) and the usual formulation of the strong mixing condition is in terms of mixing rates. Our weak invariance principle (Theorem 1 below) assumes a certain polynomial rate for the convergence of $a_{n}$ to 0 , while $\lambda_{n}$ can tend to 0 arbitrarily slowly. No assumption is made there on the rate of convergence of $\alpha(n)$ to 0 ; this rate may be essentially as slow as that of $\lambda_{n}$. This will be shown in Theorem 3 below. That theorem and the comments following it indicate that our Theorems 1 and 2 extend the class of strongly mixing stationary sequences that are known to satisfy weak or strong invariance principles.

The idea of looking at conditions (1.1) and (1.2), and "restricted $\rho$-mixing" conditions in general, arose from discussions with E. Presutti concerning the asymptotic behavior of a particle interacting with a semi-infinite ideal gas in a Bernoulli flow. (See Boldrighini, De Massi, Nogueira, and Presutti [3].)

For a given strictly stationary sequence $\left\{X_{k}\right\}$ of random variables, we shall define for each $n \geqslant 0$ the partial sum

$$
S_{n}:=X_{1}+\cdots+X_{n}
$$

where $S_{0}:=0$. If $E X_{0}=0$ and $E X_{0}^{2}<\infty$ then for each $n \geqslant 1$ the (non-negative) number $\sigma_{n}$ will be defined by

$$
\sigma_{n}^{2}=E S_{n}^{2} \text {. }
$$

For each $n=1,2,3, \ldots$, provided $\sigma_{n}>0$, define the process $\left\{W_{n}(t), 0 \leqslant t \leqslant 1\right\}$ to be the random continuous polygonal line on $[0,1]$ with vertices at the points $t=0$, $1 / n, 2 / n, \ldots, 1$ where

$$
W_{n}(k / n)=\sigma_{n}^{-1} S_{k} \quad \forall k=0,1,2, \ldots, n .
$$

Our first theorem will be a weak invariance principle under finite $(2+\delta)$ th moments $(\delta>0)$ and equation (1.2) with a certain polynomial rate of decay for $\left\{a_{n}\right\}$ and an arbitrarily slow rate of decay for $\left\{\lambda_{n}\right\}$. The main point here is that for a given $\delta$ there exists a polynomial rate on $\left\{a_{n}\right\}$ which is sufficiently fast. Our rate depends on our technique of proof; perhaps it can be improved. Our rate can be expressed as follows: First, for each $\delta \in(0,1]$, define the function $G_{\delta}:(0, \delta /(8+2 \delta)) \rightarrow(0, \infty)$ by

$$
G_{\delta}(x):=\frac{\log _{2}\left[\frac{120}{2^{(2+\delta / 2)(1 / 2-x)}-2}\right]}{x \cdot \delta^{2} /\left[2(2+\delta)^{2}\right]} .
$$


It is easy to see that, for each $\delta \in(0,1], G_{\delta}$ is positive and continuous on $(0, \delta /(8+2 \delta))$ and $G_{\delta}(x) \rightarrow \infty$ as $x$, taking values within this interval, approaches either endpoint. For each $\delta \in(0,1]$ define the number

$$
g(\delta):=\min _{0<x<\delta /(8+2 \delta)} G_{\delta}(x) .
$$

The polynomial rate of decay imposed on $\left\{a_{n}\right\}$ will be as follows:

$$
\exists \theta>g(\delta) \text { such that } a_{n} \ll n^{-\theta} \text { as } n \rightarrow \infty \text {. }
$$

In this paper the symbol $\ll$ means $\mathrm{O}(\cdot)$.

We shall also impose a condition on the variances of the partial sums, namely

$$
\forall \varepsilon>0 \quad \limsup _{n \rightarrow \infty} \frac{\sigma_{n}^{2}}{n^{1-\varepsilon}}>0 .
$$

With a different proof, perhaps it would be possible to replace (1.6) by simply $\sigma_{n}^{2} \rightarrow \infty$ as $n \rightarrow \infty$.

Theorem 1. Suppose $\left\{X_{k}\right\}$ is a strictly stationary sequence of random variables with $E X_{0}=0$. Suppose that $0<\delta \leqslant 1, E\left|X_{0}\right|^{2+\delta}<\infty$, and (1.6) holds. Suppose also that $\left\{X_{k}\right\}$ satisfies condition (1.2) with $\left\{a_{n}\right\}$ satisfying (1.5) and $\lambda_{n} \rightarrow 0$. Then, as $n \rightarrow \infty$, the process $\left\{W_{n}(t), 0 \leqslant t \leqslant 1\right\}$ converges weakly to a standard Wiener process $\{W(t), 0 \leqslant t \leqslant 1\}$.

Theorem 1 still holds if for each $n$ the process $W_{n}$ is replaced by the corresponding "step process" $\left\{\sigma_{n}^{-1} S_{[n t]}, 0 \leqslant t \leqslant 1\right\}$, where $[x]$ denotes the greatest integer $\leqslant x$. One can see this as a corollary of Theorem 1 itself, by an easy argument using Theorem 4.1 on p. 25 of Billingsley [2] (and one step in the proof of Theorem 1).

Theorem 2. Suppose that the hypothesis of Theorem 1 holds, along with the extra assumption that $\exists \lambda>1+6 / \delta$ such that $\lambda_{n} \ll(\log n)^{-\lambda}$ as $n \rightarrow \infty$. Then there exists $\sigma^{2}$, $0<\sigma^{2}<\infty$, such that $\lim _{n \rightarrow \infty} n^{-1} \sigma_{n}^{2}=\sigma^{2}$; and without changing its distribution the process $\left\{S_{n}, n \in \mathbb{N}\right\}$ can be redefined on another probability space, together with a standard Wiener process $\{W(t), t \geqslant 0\}$, such that, as $n \rightarrow \infty$,

$$
\left|S_{n}-W\left(\sigma^{2} n\right)\right|=o\left(n^{1 / 2}(\log \log n)^{-1 / 2}\right) \quad \text { a.s. }
$$

From this almost sure invariance principle one has the law of the iterated logarithm and some other results as corollaries; see Chapter 1 of Philipp and Stout [23].

Our final result will describe a class of examples.

Theorem 3. Suppose $\left\{a_{n}\right\}$ and $\left\{\lambda_{n}\right\}$ are each a non-increasing sequence of positive numbers such that $a_{n} \leqslant \lambda_{n}$ for all $n$, and as $n \rightarrow \infty, \lambda_{n} \rightarrow 0$ and $a_{n} \ll a_{2 n}$. Then there 
exists a strictly stationary sequence $\left\{X_{k}\right\}$ of random variables such that the following four statements hold:

(i) For all $n$ sufficiently large, one has that

$$
\forall A \in \mathscr{F}_{-w}^{0} \forall B \in \mathscr{F}_{n}^{\infty} \quad|P(A \cap B)-P(A) P(B)| \leqslant a_{n}+\lambda_{n}[P(A) P(B)]^{1 / 2} .
$$

(ii) $\lambda_{n} \ll \alpha(n) \ll \lambda_{n}$ as $n \rightarrow \infty$.

(iii) $\rho(n)=1$ for all $n \geqslant 1$.

(iv) If $\left\{a_{n}^{*}\right\}$ and $\left\{\lambda_{n}^{*}\right\}$ are sequences of positive numbers such that either

(a) $a_{n}^{*} \leqslant \lambda_{n}^{*} \forall n \geqslant 1$ and $\lambda_{n}^{*}=o\left(\lambda_{n}\right)$ as $n \rightarrow \infty$, or

(b) $a_{n}^{*}=\mathrm{o}\left(a_{n}\right)$ and $\lambda_{n}^{*} \rightarrow 0$ as $n \rightarrow \infty$,

then for all $n$ sufficiently large there exist $A \in \mathscr{F}_{-\infty}^{0}$ and $B \in \mathscr{F}_{n}^{\infty}$ such that

$$
|P(A \cap B)-P(A) P(B)|>a_{n}^{*}+\lambda_{n}^{*}[P(A) P(B)]^{1 / 2} .
$$

Several comments will be made in connection with Theorem 3 .

The restriction $a_{n} \ll a_{2 n}$ in Theorem 3 is of course consistent with the polynomial rate of decay of $\left\{a_{n}\right\}$ used in Theorems 1 and 2 .

The restriction $a_{n} \leqslant \lambda_{n} \forall n$ is quite reasonable. Consider for a moment the case where $a_{n} \geqslant \lambda_{n} \forall n$. Then it is obvious that strong mixing with rate $\alpha(n) \leqslant a_{n} \forall n$ implies (1.2) and that (1.2) would imply strong mixing with rate $\alpha(n) \leqslant 2 a_{n} \forall n$. Thus there would be practically no difference between (1.2) and the assumption of strong mixing with rate $\alpha(n) \ll a_{n}$ as $n \rightarrow \infty$. Thus, leaving aside the cases where the sequences $\left\{a_{n}\right\}$ and $\left\{\lambda_{n}\right\}$ are "incomparable" with each other, the only interesting cases are those in which $a_{n} \leqslant \lambda_{n} \forall n$ or rather even $a_{n}=o\left(\lambda_{n}\right)$ as $n \rightarrow \infty$.

If $\left\{X_{k}\right\}$ is a strictly stationary sequence, $\left\{a_{n}\right\}$ and $\left\{\lambda_{n}\right\}$ are sequences of positive numbers converging to 0 , and (1.2) holds, then one might have hoped that (1.2) would still hold if one judiciously changes the "balance" between the two sequences $\left\{a_{n}\right\}$ and $\left\{\lambda_{n}\right\}$, replacing either one by an appropriate "smaller" sequence and the other by a "larger" sequence (still converging to 0 ). But Theorem 3 (iv) shows that in general this cannot be done effectively either way, at least if one sticks to the requirement $a_{n} \leqslant \lambda_{n}$ (see the preceding paragraph).

In the standard earlier weak invariance principles under the mixing conditions discussed here, either strong mixing is assumed with a polynomial mixing rate (depending on the moments assumed), as in Davydov [9], Oodaira and Yoshihara [20], and Herrndorf [15], or $\rho$-mixing is assumed, sometimes with a logarithmic mixing rate, as in Ibragimov [17], Peligrad [21], and Herrndorf [16], or else strong mixing is assumed together with a small positive limit for $\rho(n)$, as in Peligrad [22]. Theorem 3 shows that Theorem 1 is not contained in any of these earlier results. Theorem 1 contains Ibragimov's classic weak invariance principle for $\rho$-mixing sequences with finite $(2+\delta)$-th moments [17, Theorem 3.1], but it does not contain the corresponding classic result for strongly mixing sequences. This last result uses the mixing rate $\Sigma \alpha(n)^{\delta /(2+\delta)}<\infty$ (see [20]), while Theorem 1 uses a faster rate on 
$\left\{a_{n}\right\}$. It would be interesting if Theorem 1 could be improved in such a way as to also contain this classic result under strong mixing.

Theorem 1 will be proved in Section 2 . Theorem 2 will be proved at the very end of Section 2. Theorem 3 will be proved in Section 3.

\section{Proof of Theorems 1 and 2}

The first three lemmas will be devoted to converting (1.2), which is a condition on events, into related conditions on random variables having certain moments.

Lemma 1. Suppose $\mathscr{A}$ and $\mathscr{B}$ are $\sigma$-fields, $a>0, \lambda>0$, and

$$
\forall A \in \mathscr{A} \quad \forall B \in \mathscr{B} \quad|P(A \cap B)-P(A) P(B)| \leqslant a+\lambda \cdot[P(A) P(B)]^{1 / 2} .
$$

Then

$$
\begin{aligned}
& \forall A \in \mathscr{A} \quad \forall B \in \mathscr{B} \quad|P(A \cap B)-P(A) P(B)| \\
& \leqslant a+2 \lambda \cdot\left[P(A) \cdot P\left(A^{c}\right) \cdot P(B) \cdot P\left(B^{c}\right)\right]^{1 / 2}
\end{aligned}
$$

Proof. Suppose $A \in \mathscr{A}$ and $B \in \mathscr{B}$. Define the events $A_{1}$ and $B_{1}$ as follows:

$$
A_{1}:=\left\{\begin{array}{ll}
A & \text { if } P(A) \leqslant \frac{1}{2}, \\
A^{\mathrm{c}} & \text { if } P(A)>\frac{1}{2} ;
\end{array} \quad B_{1}:= \begin{cases}B & \text { if } P(B) \leqslant \frac{1}{2}, \\
B^{\mathrm{c}} & \text { if } P(B)>\frac{1}{2} .\end{cases}\right.
$$

Then, by simple calculations,

$$
\begin{aligned}
& |P(A \cap B)-P(A) P(B)|=\left|P\left(A_{1} \cap B_{1}\right)-P\left(A_{1}\right) P\left(B_{1}\right)\right| \\
& \quad \leqslant a+\lambda \cdot\left[P\left(A_{1}\right) P\left(B_{1}\right)\right]^{1 / 2} \\
& \quad \leqslant a+2 \lambda \cdot\left[P\left(A_{1}\right) \cdot P\left(A_{1}^{\mathrm{c}}\right) \cdot P\left(B_{1}\right) \cdot P\left(B_{1}^{\mathrm{c}}\right)\right]^{1 / 2} . \\
& \quad=a+2 \lambda \cdot\left[P(A) \cdot P\left(A^{\mathrm{c}}\right) \cdot P(B) \cdot P\left(B^{\mathrm{c}}\right)\right]^{1 / 2} .
\end{aligned}
$$

Lemma 2. Suppose the hypothesis of Lemma 1 holds and $f \in \mathscr{L}_{\infty}(\mathscr{A}), g \in \mathscr{L}_{\infty}(\mathscr{B})$, and $E f=E g=0$. Then $|E f g| \leqslant 4 a\|f\|_{\infty}\|g\|_{\infty}+13 \cdot(2 \lambda)^{1 / 31}\|f\|_{2}\|g\|_{2}$.

Proof. Our argument will be similar to the proof of Theorem 1 of Bradley [6]; we shall indicate the differences. The first change is that the value of $t$ in [6] is replaced by $t=2 \lambda$. Now $t>0$ ( since $\lambda>0$ by assumption). If $t \geqslant 13^{-31}$ then $13 t^{1 / 31} \geqslant 1$ and Lemma 2 follows from Cauchy's inequality. Henceforth we assume (as in [6]),

$$
0<t<13^{-31} \text {. }
$$

As in [6] we can assume without loss of generality that $f$ and $g$ are mean-zero non-trivial simple functions and (replacing $f$ by $-f$ if necessary) that $E f g \geqslant 0$. Our task is to prove (instead of equation (2) of [6]) that

$$
E f g \leqslant 4 a\|f\|_{\infty}\|g\|_{\infty}+13 t^{1 / 31}\|f\|_{2}\|g\|_{2} \text {. }
$$


We represent $f$ and $g$ exactly as in [6], and in particular eqn. (3) on page 169 there remains valid. Continuing to use the notations in that reference, on page 169 , lines 16-17 there, instead of $E V_{i} W_{j} \leqslant H\left(c_{i}, d_{j}\right)$ we have $E V_{i} W_{j} \leqslant a+H\left(c_{i}, d_{j}\right)$, and instead of $E f g \leqslant \sum_{i=1}^{l-1} \sum_{j=1}^{J-1} q_{i} r_{j} H\left(c_{i}, d_{j}\right)$ we have

$$
E f g \leqslant \sum_{i=1}^{I-1} \sum_{j=1}^{J-1} q_{i} r_{j}\left[a+H\left(c_{i}, d_{j}\right)\right] .
$$

Now $\sum_{i=1}^{I-1} q_{i}=f_{I}-f_{1} \leqslant 2\|f\|_{\infty}$ and $\sum_{j=1}^{J-1} r_{j}=g_{J}-g_{1} \leqslant 2\|g\|_{\infty}$, and hence

$$
E f g \leqslant 4 a\|f\|_{\infty}\|g\|_{\infty}+\sum_{i=1}^{I-1} \sum_{j=1}^{J-1} q_{i} r_{j} H\left(c_{i}, d_{j}\right) .
$$

All that remains now is to prove that

$$
\sum_{i=1}^{I-1} \sum_{j=1}^{J-1} q_{i} r_{j} H\left(c_{i}, d_{j}\right) \leqslant 13 t^{1 / 31}\|f\|_{2}\|g\|_{2} .
$$

To do this, we simply use the argument in $[6$, page 169 , line -8 to page 170 , line $11]$ (omit the $\geq E f g$ in page 170 , line 3 there), ending with an application of Lemma 0 there. This completes the proof of Lemma 2 here.

Remark. With a bit of extra work, one might be able to replace the term $13 \cdot(2 \lambda)^{1 / 31}$ in Lemma 2 by $C(\varepsilon) \cdot \lambda^{1-\varepsilon}$ or even by $C \lambda(1-\log \lambda)$, by adapting arguments in Bulinskii [8] or Bradley and Bryc [7, Theorem 4.1(vi)], or by efficiently using a classic equality of $W$. Hoeffding,

$$
\begin{aligned}
& E X Y-E X E Y=\int_{-\infty}^{\infty} \int_{-\infty}^{\infty}[P(X \leqslant x, Y \leqslant y) \\
& -P(X \leqslant x) P(Y \leqslant y)] \mathrm{d} x \mathrm{~d} y .
\end{aligned}
$$

(For this equality see Lehmann [19, p. 1139, Lemma 2]. The argument in Bradley [6] is related to Hoeffding's equality and to arguments in Lehmann's paper.) Lemma 2 in its present form is adequate for our purposes.

Lemma 2 is a variant of a well known inequality in Ibragimov and Linnik [18, Theorem 17.2.1] involving the strong mixing coefficients $\alpha(n)$. The next lemma will be a similar variant of a result of Davydov [10, Lemma 7].

Lemma 3. Suppose the hypothesis of Lemma 1 holds and $1<p \leqslant \infty, 1<q \leqslant \infty$, $1 / p+1 / g<1, f \in \mathscr{L}_{\max \{p, 2\}}(\mathscr{A})$, and $g \in \mathscr{L}_{\max \{q, 2\}}(\mathscr{B})$. Then

$$
|E f g-E f E g| \leqslant 20 a^{1-1 / p-1 / q}\|f\|_{p}\|g\|_{q}+13 \cdot(2 \lambda)^{1 / 31}\|f\|_{2}\|g\|_{2} .
$$

Proof. We shall adapt Deo's [13, Lemma 1] proof of the cited result of Davydov [10]. Let $p, q, f$, and $g$ be arbitrary but fixed, satisfying the specifications in the statement of Lemma 3 . Without loss of generality we assume that $\|f\|_{p}>0$ and $\|g\|_{q}>0$. Define the positive numbers $C:=\|f\|_{p} \cdot a^{-1 / p}$ and $D:=\|g\|_{q} \cdot a^{-1 / q}$. Define 
the r.v.'s $f_{0}, f_{1}, g_{0}$, and $g_{1}$ by $f_{0}:=f \cdot I(|f| \leqslant C), f_{1}:=f-f_{0}, g_{0}:=g \cdot I(|g| \leqslant D)$, and $g_{1}:=g-g_{0}$, where $I$ denotes the indicator function. Then

$$
|E f g-E f E g| \leqslant\left|E f_{0} g_{0}-E f_{0} E g_{0}\right|+\left|E f_{0} g_{1}-E f_{0} E g_{1}\right|+\left|E f_{1} g-E f_{1} E g\right| \text {. }
$$

Now, by Lemma 2,

$$
\begin{aligned}
& \left|E f_{0} g_{0}-E f_{0} E g_{0}\right| \\
& \quad \leqslant 4 a\left\|f_{0}-E f_{0}\right\|_{\infty}\left\|g_{0}-E g_{0}\right\|_{\infty}+13 \cdot(2 \lambda)^{1 / 31}\left\|f_{0}-E f_{0}\right\|_{2}\left\|g_{0}-E g_{0}\right\|_{2} \\
& \quad \leqslant 16 a C D+13 \cdot(2 \lambda)^{1 / 31}\left\|f_{0}\right\|_{2}\left\|g_{0}\right\|_{2} \\
& \quad \leqslant 16 a^{1-1 / p-1 / q}\|f\|_{p}\|g\|_{q}+13 \cdot(2 \lambda)^{1 / 31}\|f\|_{2}\left\|_{g}\right\|_{2} .
\end{aligned}
$$

To estimate the second term in the R.H.S. of equation (2.3), we have

$$
\left|E f_{0} g_{1}-E f_{0} E g_{1}\right| \leqslant 2 \cdot\left\|f_{0}\right\|_{p}\left\|g_{1}\right\|_{p^{\prime}} \leqslant 2 \cdot\|f\|_{p}\left\|g_{1}\right\|_{p^{\prime}}
$$

by an application of Hölder's inequality, where $p^{\prime}$ is defined by $1 / p+1 / p^{\prime}=1$. (Hence $p^{\prime}<q$.) Now

$$
\begin{aligned}
E\left|g_{1}\right|^{p^{\prime}} & =\int_{\{|g|>D\}}|g|^{p^{\prime}} \mathrm{d} P \leqslant D^{p^{\prime}-q} \int_{\{|g|>D\}}|g|^{q} \mathrm{~d} P \\
& \leqslant D^{p^{\prime}-q}\|g\|_{q}^{q}
\end{aligned}
$$

and hence $\left\|g_{1}\right\|_{p^{\prime}} \leqslant D^{1-q / p^{\prime}}\|g\|_{q}^{q / p^{\prime}}$. Hence by equation (2.5) and simple arithmetic,

$$
\left|E f_{0} g_{1}-E f_{0} E g_{1}\right| \leqslant 2\|f\|_{p} \cdot D^{1-q / p^{\prime}}\|g\|_{q}^{q / p^{\prime}}=2 a^{1-1 / p-1 / q}\|f\|_{p}\|g\|_{q} .
$$

By a similar argument, the third term on the R.H.S. of (2.3) can be estimated by

$$
\left|E f_{1} g-E f_{1} E g\right| \leqslant 2 a^{1-1 / p-1 / q}\|f\|_{p}\|g\|_{q} .
$$

By plugging equations (2.4), (2.6) and (2.7) into (2.3), we obtain Lemma 3.

Now we turn our attention to the proof of Theorem 1. The main task in this proof is to show that under the hypothesis of Theorem $1, \sup _{n \geqslant 1}\left\|S_{n}\right\|_{2+\delta / 2} / \sigma_{n}<\infty$. The next six lemmas are devoted to proving this inequality, and the argument will be an adaptation of a well known argument of Ibragimov [17, lemma 2.1]. Once that inequality is proved, Theorem 1 (and also Theorem 2) will follow fairly quickly.

In order to simplify our use of Lemma 3 , let us introduce the notation

$$
\forall n \geqslant 1 \quad b_{n}:=13 \cdot\left(2 \lambda_{n}\right)^{1 / 31}
$$

where $\left\{\lambda_{n}\right\}$ is as in the hypothesis of Theorem 1. Note that under the hypothesis of Theorem $1, b_{n} \rightarrow 0$ as $n \rightarrow \infty$. In what follows, under the hypothesis of Theorem 1 , we assume without loss of generality that

$$
\forall n \geqslant 1 \quad a_{n} \leqslant 1 \text { and } b_{n} \leqslant 1 .
$$


Lemma 4. Suppose the hypothesis of Theorem 1 is satisfied. Then for each $\eta>0$ there exists a positive constant $C_{\eta}$ (a function of only $\eta$ and $\delta$ ) such that $\forall n \geqslant 1 \forall k \geqslant 1$ $\forall \delta^{*}, \delta^{* *}$ such that $\delta / 2 \leqslant \delta^{*}<\delta^{* *} \leqslant \delta$,

$$
\begin{aligned}
\left\|S_{2 n}\right\|_{2+\delta^{*}} \leqslant & \left\|S_{n}\right\|_{2+\delta^{* *}} \cdot\left[2+120 a_{k+1}^{\delta\left(\delta^{* *}-\delta^{*}\right) /(2+\delta)^{2}}\right. \\
& \left.+6 b_{k+1}^{\delta / 3}+\eta\right]^{1 /\left(2+\delta^{*}\right)}+C_{\eta} \sigma_{n}+2 k \cdot\left\|X_{0}\right\|_{2+\delta} .
\end{aligned}
$$

Proof. First let us suppose that $n \geqslant 1, k \geqslant 1$, and $\delta / 2 \leqslant \delta^{*}<\delta^{* *} \leqslant \delta$, and let us carry out some calculations. Define the notation $\bar{S}_{n}:=S_{2 n+k}-S_{n+k}$. Then by Minkowski's inequality,

$$
\begin{aligned}
\left\|S_{2 n}\right\|_{2+\delta^{*}} & \leqslant\left\|S_{n}+\bar{S}_{n}\right\|_{2+\delta^{*}}+\left\|S_{n+k}-S_{n}\right\|_{2+\delta^{*}}+\left\|S_{2 n+k}-S_{2 n}\right\|_{2+\delta^{*}} \\
& \leqslant\left\|S_{n}+\bar{S}_{n}\right\|_{2+\delta^{*}}+2 k \cdot\left\|X_{0}\right\|_{2+\delta} .
\end{aligned}
$$

Now let us estimate the term $E\left|S_{n}+\bar{S}_{n}\right|^{2+\delta^{*}}$. Recall that $\delta \leqslant 1$ by hypothesis.

$$
\begin{aligned}
E\left|S_{n}+\bar{S}_{n}\right|^{2+\delta^{*}} \leqslant & E\left(S_{n}+\bar{S}_{n}\right)^{2}\left(\left|S_{n}\right|^{\delta^{*}}+\left|\bar{S}_{n}\right|^{\delta^{*}}\right) \\
\leqslant & 2 E\left|S_{n}\right|^{2+\delta^{*}}+E\left|S_{n}\right|^{\delta^{*}}\left|\bar{S}_{n}\right|^{2}+E\left|S_{n}\right|^{2}\left|\bar{S}_{n}\right|^{\delta^{*}} \\
& +2 E\left|S_{n}\right| \cdot\left|\bar{S}_{n}\right|^{1+\delta^{*}}+2 E\left|S_{n}\right|^{1+\delta^{*}}\left|\bar{S}_{n}\right| \\
\leqslant & 2 E\left|S_{n}\right|^{2+\delta^{*}}+3 E\left|S_{n}\right|^{\delta^{*}}\left|\bar{S}_{n}\right|^{2}+3 E\left|S_{n}\right|^{2}\left|\bar{S}_{n}\right|^{\delta^{*}}
\end{aligned}
$$

where the last inequality comes from the elementary fact that if $x \geqslant 0$ and $y \geqslant 0$ are real numbers then $x y^{1+\delta^{*}}+x^{1+\delta^{*}} y \leqslant x^{\delta^{*}} y^{2}+x^{2} y^{\delta^{*}}$.

Next let us estimate $E\left|S_{n}\right|^{\delta^{*}}\left|\bar{S}_{n}\right|^{2}$. We shall apply Hölder's inequality, and Lemma 3 (and (2.8)) with $p=q=2\left(2+\delta^{* *}\right) /\left(2+\delta^{*}\right)$, and (2.9).

$$
\begin{aligned}
& E\left|S_{n}\right|^{\delta^{*}}\left|\bar{S}_{n}\right|^{2} \\
& \leqslant\left[E\left|\bar{S}_{n}\right|^{2+\delta^{*}}\right]^{\left(2-\delta^{*}\right) /\left(2+\delta^{*}\right)} \cdot\left[E\left|S_{n} \bar{S}_{n}\right|^{\left(2+\delta^{*}\right) / 2}\right]^{2 \delta^{*} /\left(2+\delta^{*}\right)} \\
& \leqslant\left\|\bar{S}_{n}\right\|_{2+\delta^{*}}^{2-\delta^{*}} \cdot\left[20 a_{k+1}^{1-1 / p-1 / q}\left\|\left|\bar{S}_{n}\right|^{\left(2+\delta^{*}\right) / 2}\right\|_{p} \cdot\left\|\left|S_{n}\right|^{\left(2+\delta^{*}\right) / 2}\right\|_{q}\right. \\
&+b_{k+1}\left\|\left|\bar{S}_{n}\right|^{\left(2+\delta^{*}\right) / 2}\right\|_{2} \cdot\left\|\left|S_{n}\right|^{\left(2+\delta^{*}\right) / 2}\right\|_{2} \\
&\left.+E\left|\bar{S}_{n}\right|^{\left(2+\delta^{*}\right) / 2} E\left|S_{n}\right|^{\left(2+\delta^{*}\right) / 2}\right]^{2 \delta^{*} /\left(2+\delta^{*}\right)} \\
&=\left\|S_{n}\right\|_{2+\delta^{*}}^{2-\delta^{*}} \cdot\left[20 a_{k+1}^{\left(\delta^{* *}-\delta^{*}\right) /\left(2+\delta^{* *}\right)}\left\|S_{n}\right\|_{2+\delta^{* *}}^{2+\delta^{*}}\right. \\
&\left.+b_{k+1}\left\|S_{n}\right\|_{2+\delta^{*}}^{2+\delta^{*}}+\left\|S_{n}\right\|_{\left(2+\delta^{*}\right) / 2}^{2+\delta^{*}}\right]^{2 \delta^{*} /\left(2+\delta^{*}\right)} \\
& \leqslant\left\|S_{n}\right\|_{2+\delta^{* *}}^{2-\delta^{*}} \cdot\left[20 a_{k+1}^{\left(\delta^{* *}-\delta^{*}\right) /\left(2+\delta^{* *}\right)}\left\|S_{n}\right\|_{2+\delta^{* *}}^{2+\delta^{*}}\right. \\
&\left.+b_{k+1}\left\|S_{n}\right\|_{2+\delta^{* *}}^{2+\delta^{*}}+\left\|S_{n}\right\|_{2}^{2+\delta^{*}}\right]^{2 \delta^{*} /\left(2+\delta^{*}\right)} \\
& \leqslant\left\|S_{n}\right\|_{2+\delta^{* * *}}^{2-\delta^{*}} \cdot\left[20 a_{k+1}^{2 \delta^{*}\left(\delta^{* *}-\delta^{*}\right) /\left[\left(2+\delta^{*}\right)\left(2+\delta^{* *}\right)\right]}\left\|S_{n}\right\|_{2+\delta^{* *}}^{2 \delta^{*}}\right. \\
&\left.+b_{k+1}^{2 \delta^{*} /\left(2+\delta^{*}\right)}\left\|S_{n}\right\|_{2+\delta^{* *}}^{2 \delta^{*}}+\left\|S_{n}\right\|_{2}^{2 \delta^{*}}\right] \\
& \leqslant\left\|S_{n}\right\|_{2+\delta^{* *}}^{2+\delta^{*}} \cdot\left[20 a_{k+1}^{\delta\left(\delta^{* *}-\delta^{*}\right) /(2+\delta)^{\prime}}+b_{k+1}^{\delta / 3}\right]+\left\|S_{n}\right\|_{2+\delta^{* *}}^{2-\delta^{*}}\left\|S_{n}\right\|_{2}^{2 \delta^{*}}
\end{aligned}
$$


The same estimate holds for $E\left|S_{n}\right|^{2}\left|\vec{S}_{n}\right|^{\delta^{*}}$, and hence by (2.11) we have that

$$
\begin{aligned}
E\left|S_{n}+\bar{S}_{n}\right|^{2+\delta^{*}} \leqslant & \left\|S_{n}\right\|_{2+\delta^{* *}}^{2+\delta^{*}}\left[2+120 a_{k+1}^{\delta\left(\delta^{* *}-\delta^{*}\right) /(2+\delta)^{2}}+6 b_{k+1}^{\delta / 3}\right] \\
& +6\left\|S_{n}\right\|_{2+\delta^{* *}}^{2-\delta^{*}}\left\|S_{n}\right\|_{2}^{2 \delta^{*}} .
\end{aligned}
$$

In proving Lemma 4 it suffices to consider only values of $\eta \in(0,1]$. Henceforth we impose this restriction on $\eta$. For each $\eta \in(0,1]$ define the constant $C_{\eta}$ by $C_{\eta}:=6^{(2+\delta) / \delta} \eta^{-2 / \delta}$. Then for each such $\eta, C_{\eta} \geqslant 6^{\left(2+\delta^{*}\right) /\left(2 \delta^{*}\right)} \eta^{-\left(2-\delta^{*}\right) /\left(2 \delta^{*}\right)}$ and, by simple arithmetic, $\left(6 / C_{\eta}\right)^{\left.1,12 \delta^{*}\right)} \leqslant(\eta / 6)^{1 /\left(2 \delta^{*}\right)}$. Hence either

(i) $\sigma_{n} /\left\|S_{n}\right\|_{2+\delta^{* *}} \leqslant(\eta / 6)^{1 /\left(2 \delta^{*}\right)}$ or

(ii) $\sigma_{n} /\left\|S_{n}\right\|_{2+\delta^{* *}} \geqslant\left(6 / C_{\eta}\right)^{1 /\left(2-\delta^{*}\right)}$.

If (i) holds then, by simple arithmetic, $6\left\|S_{n}\right\|_{2+\delta^{* *}}^{2-\delta^{*}} \sigma_{n}^{2 \delta^{*}} \leqslant \eta \cdot\left\|S_{n}\right\|_{2+\delta^{* *}}^{2+\delta^{*}}$. If (ii) holds then, by simple arithmetic, $6\left\|S_{n}\right\|_{2+\delta^{* *}}^{2-\delta^{*}} \sigma_{n}^{2 \delta^{*}} \leqslant C_{\eta} \sigma_{n}^{2+\delta^{*}}$. In either case, by (2.12), for each $\eta \in(0,1]$,

$$
E\left|S_{n}+\bar{S}_{n}\right|^{2+\delta^{*}} \leqslant\left\|S_{n}\right\|_{2+\delta^{* *}}^{2+\delta^{*}}\left[2+120 a_{k+1}^{\delta\left(\delta^{* *}-\delta^{*}\right) /(2+\delta)^{2}}+6 b_{k+1}^{\delta / 3}+\eta\right]+C_{\eta} \sigma_{n}^{2+\delta^{*}}
$$

and hence (using the trivial fact that $C_{\eta}>1$ )

$$
\left\|S_{n}+\bar{S}_{n}\right\|_{2+\delta^{*}} \leqslant\left\|S_{n}\right\|_{2+\delta^{* *}}\left[2+120 a_{k+1}^{\delta\left(\delta^{* *}-\delta^{*}\right) /(2+\delta)^{2}}+6 b_{k+1}^{\delta / 3}+\eta\right]^{1 /\left(2+\delta^{*}\right)}+C_{\eta} \sigma_{n} .
$$

Since $C_{\eta}$ depends only on $\eta$ and $\delta$, Lemma 4 follows from (2.10).

In what follows, expressions such as $2^{x}$ will frequently occur as subscripts. For typographical convenience we shall use the following notation:

$$
\forall x \in \mathbb{R} \quad \operatorname{doub}(x):=2^{x} .
$$

When necessary we shall use the notation $[x]:=$ greatest integer $\leqslant x$.

Also, if $r=0$ then $\sum_{i=1}^{r}$ (anything) is interpreted to be 0 .

Lemma 5. Suppose the hypothesis of Theorem 1 is satisfied. Then $\exists \beta>0 \exists A>0$ such that $\forall r \in\{0,1,2, \ldots\}$,

$$
\left\|S_{\mathrm{doub}(r)}\right\|_{2+\delta / 2} \leqslant 2^{r(1 / 2-\beta)}\left\|X_{0}\right\|_{2+\delta}+A \cdot\left(\sum_{i=1}^{r} 2^{(i-1)(1 / 2-\beta)} \sigma_{\mathrm{doub}(r-i)}\right) .
$$

Proof. Using (1.5) we fix $\theta>g(\delta)$ such that

$$
a_{n}=o\left(n^{-\theta}\right) \text { as } n \rightarrow \infty \text {. }
$$

Using (1.3), (1.4) we fix $\gamma, 0<\gamma<\delta /(8+2 \delta)$, such that $\theta>G_{\delta}(\gamma)$. Then we fix $\varepsilon$, $0<\varepsilon<\gamma$, such that

$$
\theta>\frac{\log _{2}\left[\frac{120}{2^{(2+\delta / 2)(1 / 2-\gamma)}-2}\right]}{\varepsilon \delta^{2} /\left[2(2+\delta)^{2}\right]} .
$$


By elementary arithmetic,

$$
2^{-\theta \varepsilon \delta^{2} /\left[2(2+\delta)^{2}\right]}<120^{-1} \cdot\left(2^{(2+\delta / 2)(1 / 2-\gamma)}-2\right)
$$

and hence

$$
2+120 \cdot 2^{-\theta \varepsilon \delta^{2} /\left[2(2+\delta)^{2}\right]}<2^{(2+\delta / 2)(1 / 2-\gamma)} .
$$

By (2.13), $a_{n}<n^{-\theta}$ for all $n$ sufficiently large. Hence for all positive integers $r$ sufficiently large,

$$
a_{[\mathrm{doub}(r \varepsilon)]+1}^{\delta^{2} /\left[2 r(2+\delta)^{2}\right]}<2^{-\theta \varepsilon \delta^{2} /\left[2(2+\delta)^{2}\right]}
$$

and hence

$$
2+120 \cdot a_{[\operatorname{doub}(r \varepsilon)]+1}^{\delta^{2} /\left[2 r(2+\delta)^{2}\right]}<2^{(2+\delta / 2)(1 / 2-\gamma)} .
$$

Fix $\mu, \varepsilon<\mu<\gamma$. Fix $\eta, 0<\eta<2^{(2+\delta / 2)(1 / 2-\mu)}-2^{(2+\delta / 2)(1 / 2-\gamma)}$. Since $b_{k} \rightarrow 0$ as $k \rightarrow \infty$, one has that, for all integers $r$ sufficiently large,

$$
6 b_{[\mathrm{doub}(r \varepsilon)]+1}^{\delta / 3}+\eta<2^{(2+\delta / 2)(1 / 2-\mu)}-2^{(2+\delta / 2)(1 / 2-\gamma)} .
$$

Hence, for all integers $r$ sufficiently large,

$$
\left(2+120 \cdot a_{[\operatorname{doub}(r \varepsilon)]+1}^{\delta^{2}\left[2 r(2+\delta)^{2}\right]}+6 b_{[\operatorname{doub}(r \varepsilon)]+1}^{\delta / 3}+\eta\right)^{1 /(2+\delta / 2)}<2^{1 / 2-\mu} .
$$

Fix $\beta$ such that

$$
0<\beta<\mu-\varepsilon .
$$

Let us now suppose that $r$ is a positive integer so large that (2.14) holds, and let us carry out some calculations. For each $i=0,1,2, \ldots, r$ define $\delta_{i}:=\delta / 2+i \cdot \delta /(2 r)$. Then for each $i=1,2, \ldots, r, \delta\left(\delta_{i}-\delta_{i-1}\right) /(2+\delta)^{2}=\delta^{2} /\left[2 r(2+\delta)^{2}\right]$ and hence by Lemma 4 (with $k=\left[2^{r \varepsilon}\right]$ ) and (2.14),

$$
\forall n \geqslant 1 \quad\left\|S_{2 n}\right\|_{2+\delta_{i-1}} \leqslant\left\|S_{n}\right\|_{2+\delta_{i}} \cdot 2^{1 / 2-\mu}+C_{\eta} \sigma_{n}+2^{r E+1}\left\|X_{0}\right\|_{2+\delta}
$$

where $C_{\eta}$ is as in the statement of Lemma 4. Applying this repeatedly, with $(n, i)$ being $(1, r)$, then $(2, r-1)$, then $(4, r-2)$, then $(8, r-3), \ldots$ we finally obtain

$$
\begin{aligned}
\left\|S_{\mathrm{doub}(r)}\right\|_{2+\delta / 2} \leqslant & {\left[2^{r(1 / 2-\mu)}+\sum_{i=1}^{r} 2^{(i-1)(1 / 2-\mu)} \cdot 2^{r \varepsilon+1}\right] \cdot\left\|X_{0}\right\|_{2+\delta} } \\
& +C_{\eta} \cdot \sum_{i=1}^{r} 2^{(i-1)(1 / 2-\mu)} \sigma_{\mathrm{doub}(r-i)} \\
\leqslant & (2 r+1) \cdot 2^{r(1 / 2-\mu+\varepsilon)} \cdot\left\|X_{0}\right\|_{2+\delta}+C_{\eta} \cdot \sum_{i=1}^{r} 2^{(i-1)(1 / 2-\mu)} \sigma_{\mathrm{doub}(r-i)} .
\end{aligned}
$$

By (2.15), for all $r$ sufficiently large,

$$
\left\|S_{\mathrm{doub}(r)}\right\|_{2+\delta / 2} \leqslant 2^{r(1 / 2-\beta)}\left\|X_{0}\right\|_{2+\delta}+C_{\eta} \cdot \sum_{i=1}^{r} 2^{(i-1)(1 / 2-\beta)} \sigma_{\mathrm{doub}(r-i)} .
$$


By replacing $C_{\eta}$ by a larger constant if necessary, we can include the (finitely many) remaining values of $r \geqslant 1$. Thus Lemma 5 holds for all $r \geqslant 1$. Also, Lemma 5 holds for $r=0$ by the simple equation $\left\|S_{1}\right\|_{2+\delta / 2}=\left\|X_{0}\right\|_{2+\delta / 2} \leqslant\left\|X_{0}\right\|_{2+\delta}$. This completes the proof.

Lemma 6. Suppose the hypothesis of Theorem 1 is satisfied. Then $\forall \gamma>0 \exists B_{\gamma}>0$ such that $\forall i$, $r$ with $0 \leqslant i \leqslant r$, one has that $\sigma_{\mathrm{doub}(r)} \geqslant B_{\gamma} \cdot 2^{(r-i)(1 / 2-\gamma)} \sigma_{\mathrm{doub}(i)}$.

Proof. First let us suppose that $n \geqslant 1$ and $k \geqslant 0$, and let us carry out some calculations.

Let $\bar{S}_{n}$ be as in the proof of Lemma 4. Similarly to equation (2.10) there,

$$
\left\|S_{2 n}\right\|_{2} \geqslant\left\|S_{n}+\bar{S}_{n}\right\|_{2}-2 k\left\|X_{0}\right\|_{2} \text {. }
$$

Applying Lemma 3 with $p=q=2+\delta / 2$, along with (2.8), we obtain

$$
\left|\operatorname{Cov}\left(S_{n}, \bar{S}_{n}\right)\right| \leqslant 20 a_{k+1}^{\delta /(4+\delta)}\left\|S_{n}\right\|_{2+\delta / 2}^{2}+b_{k+1}\left\|S_{n}\right\|_{2}^{2}
$$

and hence

$$
\begin{aligned}
\left\|S_{n}+\bar{S}_{n}\right\|_{2} & \geqslant\left[2\left(1-b_{k+1}\right)\left\|S_{n}\right\|_{2}^{2}-40 a_{k+1}^{\delta /(4+\delta)}\left\|S_{n}\right\|_{2+\delta / 2}^{2}\right]^{1 / 2} \\
& \geqslant 2^{1 / 2}\left(1-b_{k+1}\right)\left\|S_{n}\right\|_{2}-40^{1 / 2} a_{k+1}^{\delta /(8+2 \delta)}\left\|S_{n}\right\|_{2+\delta / 2}
\end{aligned}
$$

(where $[\cdot]^{1 / 2}$ is replaced by 0 if $[\cdot]$ is negative) and hence

$$
\left\|S_{2 n}\right\|_{2} \geqslant 2^{1 / 2}\left(1-b_{k+1}\right)\left\|S_{n}\right\|_{2}-40^{1 / 2} a_{k+1}^{\delta /(8+2 \delta)}\left\|S_{n}\right\|_{2+\delta / 2}-2 k\left\|X_{0}\right\|_{2}
$$

We shall come back to (2.16) shortly. Now let $0<\beta<\frac{1}{2}$ and $A>0$ be as in Lemma 5 . Let $\mathscr{S}$ denote the set of all non-negative integers $r$ such that

$$
\sigma_{\text {doub }(r)} / 2^{r(1 / 2-\beta)} \geqslant \sigma_{\text {doub }(i)} / 2^{i(1 / 2-\beta)} \quad \forall i=0,1, \ldots, r .
$$

We need to show that $\mathscr{S}$ is an infinite set. To do this, it suffices to show that $s:=\sup _{r \geqslant 0} \sigma_{\operatorname{doub}(r)} / 2^{r(1 / 2-\beta)}=\infty$. Nuw, for any $n \geqslant 1$, defining $r$ by $2^{r} \leqslant n<2^{r+1}$ and expressing $n$ in binary form, we have that

$$
\begin{aligned}
\sigma_{n} & \leqslant \sum_{i=0}^{r} \sigma_{\mathrm{doub}(i)} \leqslant \sum_{i=0}^{r} s \cdot 2^{i(1 / 2-\beta)} \\
& =s \cdot 2^{r(1 / 2-\beta)} \cdot \sum_{j=0}^{r} 2^{-j(1 / 2-\beta)} \\
& \leqslant n^{(1 / 2-\beta)} \cdot s \cdot \sum_{j=0}^{\infty} 2^{-J(1 / 2-\beta)} .
\end{aligned}
$$

Since the last sum is finite, the assumption (1.6) (with any $\varepsilon \in(0,2 \beta)$ ) implies $s=\infty$. Hence $\mathscr{S}$ is an infinite set.

Obviously, for each $r \in \mathscr{S}$ one has that $\sigma_{\mathrm{doub}(r)} \geqslant \sigma_{\mathrm{doub}(i)} \forall i=0,1, \ldots, r$ and hence (see Lemma 5)

$$
\left\|S_{\mathrm{doub}(r)}\right\|_{2+\delta / 2} \leqslant 2^{r(1 / 2-\beta)}\left\|X_{0}\right\|_{2+\delta}+A \cdot \sigma_{\mathrm{doub}(r)} \cdot \sum_{i=1}^{r} 2^{(i-1)(1 / 2-\beta)}
$$


The sum is $\ll 2^{r(1 / 2-\beta)}$ as $r \rightarrow \infty$. We fix $B>0$ such that, for all $r \in \mathscr{P},\left\|S_{\mathrm{doub}(r)}\right\|_{2+\delta / 2} \leq$ $B \cdot 2^{r(1 / 2-\beta)} \sigma_{\text {doub }(r)}$. By $(2.16)$, for all $k \geqslant 0$ and $r$ such that $r-1 \in \mathscr{Y}$,

$$
\begin{aligned}
\sigma_{\mathrm{doub}(r)} \geqslant & 2^{1 / 2}\left(1-b_{k+1}\right) \sigma_{\mathrm{doub}(r-1)} \\
& -40^{1 / 2} B a_{k+1}^{\delta /(8+2 \delta)} \cdot 2^{(r-1)(1 / 2-\beta)} \sigma_{\mathrm{doub}(r-1)}-2 k \sigma_{1} .
\end{aligned}
$$

Referring to (1.3), (1.4), it is easy to show that $g(\delta)>(8+2 \delta) / \delta$. Using (1.5) in the hypothesis of Theorem 1 , we fix $\theta>(8+2 \delta) / \delta$ such that $a_{k} \leqslant k^{-\theta}$ as $k \rightarrow \infty$. We fix $Q>0$ such that for all $k \geqslant 0$ and $r$ such that $r-1 \in \mathscr{S}$,

$$
\begin{aligned}
\sigma_{\mathrm{doub}(r)} \geqslant & 2^{1 / 2}\left(1-b_{k+1}\right) \sigma_{\mathrm{doub}(r-1)} \\
& -Q \cdot(k+1)^{-\theta \delta /(8+2 \delta)} \cdot 2^{(r-\mathbf{i})(1 / 2-\beta)} \sigma_{\mathrm{doub}(r-1)}-2 k \sigma_{1} .
\end{aligned}
$$

Now suppose $r-1 \in \mathscr{S}$ and $\eta>0$. Taking $k=\left[\eta \cdot \sigma_{\text {doub }(r-1)}\right]$ we obtain

$$
\begin{aligned}
\sigma_{\mathrm{doub}(r)} \geqslant & 2^{1 / 2}\left(1-b_{k+1}\right) \sigma_{\mathrm{doub}(r-1)} \\
& -Q \eta^{-\theta \delta /(8+2 \delta)} \cdot \sigma_{\mathrm{doub}(r-1)}^{1-\theta \delta /(8+2 \delta)} \cdot 2^{(r-1)(1 / 2-\beta)}-2 \eta \cdot \sigma_{1} \cdot \sigma_{\mathrm{doub}(r-1)} .
\end{aligned}
$$

Now suppose $\gamma>0$. Let us fix $\eta>0$ so small that $1-2 \eta\left\|X_{0}\right\|_{2}>2^{-\gamma}$. Then as $r \rightarrow \infty$ subject to the restriction $r-1 \in \mathscr{P}, b_{k+1} \rightarrow 0$ (since $k=\left[\eta \cdot \sigma_{\mathrm{doub}(r-1)}\right] \rightarrow \infty$ ), and $\sigma_{\operatorname{doub}(r-1)}^{1-\theta \delta(8+2 \delta)} \cdot 2^{(r-1)(1 / 2-\beta)}=\mathrm{o}\left(\sigma_{\mathrm{doub}(r-1)}\right)$ (by the definition of $\mathscr{P}$ and the fact that $\theta \delta /(8+2 \delta)>1$ by choice of $\theta)$. Hence there exists $r_{0}=r_{0}(\gamma)$ such that $\forall r \geqslant r_{0}$ such that $r-1 \in \mathscr{S}, \sigma_{\text {doub }(r)} \geqslant 2^{1 / 2-\gamma} \sigma_{\text {doub }(r-1)}$. Considering some $\gamma, 0<\gamma<\beta$, one has from the definition of $\mathscr{S}$ that if $r \geqslant r_{0}(\gamma)$ and $r-1 \in \mathscr{S}$ then $r \in \mathscr{S}$. Since $\mathscr{S}$ is an infinite set, such an $r$ must exist, and by induction one has that $\mathscr{S}$ contains all but finitely many positive integers.

Now let $\gamma>0$ be arbitrary but fixed. By the preceding argument there exists $r^{*}=r^{*}(\gamma)$ such that $\forall r \geqslant r^{*} \sigma_{\text {doub }(r)} \geqslant 2^{1 / 2-\gamma} \sigma_{\text {doub(r-1) }}$. Define

$$
c:=\inf \left[\sigma_{\mathrm{doub}(r)} /\left(2^{1 / 2-\gamma} \sigma_{\mathrm{doub}(r-1)}\right)\right], \quad 1 \leqslant r \leqslant r^{*} .
$$

Define $B_{\gamma}:=1 \wedge c^{r^{*}}$. Then by a simple argument, $B_{\gamma}>0$ and $\forall i, r, 0 \leqslant i \leqslant r$, $\sigma_{\mathrm{doub}(r)} \geqslant B_{\gamma} \cdot 2^{(r-i)(1 / 2-\gamma)} \sigma_{\mathrm{doub}(i)}$. Thus Lemma 6 holds. This completes the proof.

Lemma 7. Suppose the hypothesis of Theorem 1 is satisfied. Then there exists a constant $B>0$ such that $\forall r \geqslant 0,\left\|S_{\text {doub }(r)}\right\|_{2+\delta / 2} \leqslant B \sigma_{\text {doub }(r)}$.

Proof. Fix $0<\beta<\frac{1}{2}$ and $A>0$ as in Lemma 5. Fix $\gamma, 0<\gamma<\beta$. Fix $B_{\gamma}>0$ as in Lemma 6. From Lemma 5 we have that, for each $r \geqslant 0$,

$$
\begin{aligned}
& \left\|S_{\mathrm{doub}(r)}\right\|_{2+\delta / 2} \\
& \quad \leqslant \sigma_{\mathrm{doub}(r)}\left[\frac{2^{r(1 / 2-\beta)}}{\sigma_{\mathrm{doub}(r)}}\left\|X_{0}\right\|_{2+\delta}+A \cdot \sum_{i=1}^{r}\left(2^{(i-1)(1 / 2-\beta)}-\frac{\sigma_{\mathrm{doub}(r-i)}}{\sigma_{\mathrm{doub}(r)}}\right)\right] \\
& \quad \leqslant \sigma_{\mathrm{doub}(r)}\left[\frac{2^{r(1 / 2-\beta)}}{\sigma_{\mathrm{doub}(r)}}\left\|X_{0}\right\|_{2+\delta}+A B_{\gamma}^{-1} \sum_{i=1}^{r} 2^{(i-1)(1 / 2-\beta)} \cdot 2^{-i(1 / 2-\gamma)}\right] .
\end{aligned}
$$


By Lemma 6 for our fixed $\gamma$, the term $2^{r(1 / 2-\beta)} / \sigma_{\text {doub }(r)}$ vanishes as $r \rightarrow \infty$. Also, the sum $\sum_{i=1}^{r} 2^{(i-1)(1 / 2-\beta)} \cdot 2^{-i(1 / 2-\gamma)}$ is bounded above by $\sum_{i=1}^{\infty} 2^{i(\gamma-\beta)}<\infty$. Lemma 7 follows.

Lemma 8. Suppose the hypothesis of Theorem 1 holds. Then there exists a constant $D>0$ such that $\forall r=0,1,2, \ldots, \forall n, 2^{r} \leqslant n \leqslant 2^{r+1}$, one has $\sigma_{n} \geqslant D \cdot \sigma_{\operatorname{doub}(r)}$; hence $\sigma_{n}^{2} \rightarrow \infty$ as $n \rightarrow \infty$.

Proof. Obviously we can ignore the case $n=2^{r}$ (for $D=1$ will work then). Let us suppose that $r \geqslant 0,2^{r}<n \leqslant 2^{r+1}$, and $k \geqslant 1$, and let us carry out some calculations. Define $\bar{S}_{\text {doub }(r)}:=S_{n+k}-S_{n-\operatorname{doub}(r)+k}$. Arguing as in Lemma 6 , we have

$$
\left\|S_{n}\right\|_{2} \geqslant\left\|S_{n-\operatorname{doub}(r)}+\bar{S}_{\mathrm{doub}(r)}\right\|_{2}-2 k \cdot\left\|X_{0}\right\|_{2} \text {. }
$$

Applying Lemma 3 with $p=2$ and $q=2+\delta / 2$, we have

$$
\begin{aligned}
& \left|\operatorname{Cov}\left(S_{n-\operatorname{doub}(r)}, \bar{S}_{\mathrm{doub}(r)}\right)\right| \\
& \quad \leqslant 20 a_{k}^{\delta /(8+2 \delta)}\left\|S_{n-\operatorname{doub}(r)}\right\|_{2} \cdot\left\|S_{\mathrm{doub}(r)}\right\|_{2+\delta / 2}+b_{k} \cdot\left\|S_{n-\operatorname{doub}(r)}\right\|_{2}\left\|_{\operatorname{doub}(r)}\right\|_{2}
\end{aligned}
$$

and hence

$$
\begin{aligned}
& E\left[S_{n-\operatorname{doub}(r)}+\bar{S}_{\mathrm{doub}(r)}\right]^{2} \\
& \geqslant E S_{n-\operatorname{doub}(r)}^{2}+E S_{\mathrm{doub}(r)}^{2}-2 b_{k}\left\|S_{n-\operatorname{doub}(r)}\right\|_{2}\left\|S_{\mathrm{doub}(r)}\right\|_{2} \\
&-40 a_{k}^{\delta /(8+2 \delta)}\left\|S_{n-\operatorname{doub}(r)}\right\|_{2}\left\|S_{\mathrm{doub}(r)}\right\|_{2+\delta / 2} \\
& \geqslant E S_{n-\operatorname{doub}(r)}^{2}+E S_{\mathrm{doub}(r)}^{2}-b_{k}\left(E S_{n-\operatorname{doub}(r)}^{2}+E S_{\mathrm{doub}(r)}^{2}\right) \\
&-20 a_{k}^{\delta /(8+2 \delta)}\left(E S_{n-\operatorname{doub}(r)}^{2}+\left\|S_{\mathrm{doub}(r)}\right\|_{2+\delta / 2}^{2}\right) \\
& \geqslant\left(1-b_{k}-20 a_{k}^{d /(8+2 \delta)}\right) E S_{n-\operatorname{doub}(r)}^{2}+\left(1-b_{k}-20 B^{2} a_{k}^{\delta /(8+2 \delta)}\right) E S_{\mathrm{doub}(r)}^{2}
\end{aligned}
$$

where $B$ is as in Lemma 7 .

Fix $k$ such that $1-b_{k}-20\left(\max \left\{1, B^{2}\right\}\right) a_{k}^{\delta /(8+2 \delta)} \geqslant \frac{1}{2}$. Then $\forall r \geqslant 0 \forall n 2^{r}<n \leqslant 2^{r+1}$, one has that $E\left[S_{n-\operatorname{doub}(r)}+\bar{S}_{\mathrm{doub}(r)}\right]^{2} \geqslant(1 / 2) E S_{\mathrm{doub}(r)}^{2}$; and hence, by $(2.17)$,

$$
\left\|S_{n}\right\|_{2} \geqslant(1 / 2)^{1 / 2}\left\|S_{\mathrm{doub}(r)}\right\|_{2}-2 k\left\|X_{0}\right\|_{2} \text {. }
$$

By Lemma 6, if $r$ is sufficiently large and $2^{r}<n \leqslant 2^{r+1}$ then $\left\|S_{n}\right\|_{2} \geqslant(1 / 2)\left\|S_{\text {doub }(r)}\right\|_{2}$. Replacing $1 / 2$ by a smaller positive constant if necessary, we can obtain Lemma 8 . (The second conclusion of Lemma 8 now follows from Lemma 6.)

Lemma 9. If the hypothesis of Theorem 1 is satisfied, then $\sup _{n \geqslant 1}\left\|S_{n}\right\|_{2+\delta / 2} / \sigma_{n}<\infty$.

Proof. Let the constants $B$ and $D$ be as in Lemmas 7 and 8 . Fix $0<\gamma<\frac{1}{2}$. Let $B_{\gamma}$ be as in Lemma 6. 
Let $n \geqslant 1$ be arbitrary but fixed. Define $r$ by $2^{r} \leqslant n<2^{r+1}$. Expressing $n$ in binary form, we obtain

$$
\begin{aligned}
\left\|S_{n}\right\|_{2+\delta / 2} & \leqslant \sum_{i=0}^{r}\left\|S_{\mathrm{doub}(i)}\right\|_{2+\delta / 2} \leqslant \sum_{i=0}^{r} B \cdot \sigma_{\mathrm{doub}(i)} \\
& \leqslant B \cdot \sum_{i=0}^{r} B_{\gamma}^{-1} \cdot 2^{-(r-i)(1 / 2-\gamma)} \sigma_{\mathrm{doub}(r)} \\
& \leqslant\left[B \cdot B_{\gamma}^{-1} D^{-1} \sum_{j=0}^{\infty} 2^{-j(1 / 2-\gamma)}\right] \sigma_{n} .
\end{aligned}
$$

Since the term in the brackets is a (finite) constant, Lemma 9 is proved.

Proof of Theorem 1. Recall that (1.2) implies strong mixing. By Lemma 9 and Ibragimov and Linnik [18, p. 307, Theorem 17.2.2], $\sup _{m \geqslant 0, n \geqslant 0} \mid \operatorname{Corr}\left(X_{-m}+\cdots+X_{0}\right.$, $\left.X_{I}+\cdots+X_{I+n}\right) \mid$ converges to 0 as $I \rightarrow \infty$. By Ibragimov and Linnik [18, p. 330, lines 6-14] and Lemma $8, \operatorname{Var} S_{n}=n \cdot h(n)$ where $h:(0, \infty) \rightarrow(0, \infty)$ is a slowly varying function. Hence by Lemma 9 , Ibragimov and Linnik [18, p. 397, property (3)] (for $1 / h$ as well as $h$ ), and some simple calculations,

$$
\left[\sup \frac{E\left|W_{n}(s)-W_{n}(t)\right|^{2+\delta / 2}}{|s-t|^{1 / \delta / 5}}, n \geqslant 1,0 \leqslant s<t \leqslant 1\right]<\infty .
$$

Hence in Billingsley [2, p. 157, Theorem 19.2] the tightness condition (eqn. (19.15) there) is satisfied. (See Billingsley [2], p. 95, Theorem 12.3 and eqn. (12.51) and p. 55 , Theorem 8.2.) In [2, Theorem 19.2] the uniform integrability condition is satisfied by Lemma 9. Now Theorem 1 follows from Billingsley [2, Theorem 19.2] (the rest of the conditions there are easy to check).

Proof of Theorem 2. By the hypothesis of Theorem 2, one has that $\left\{X_{k}\right\}$ is strongly mixing with mixing rate $\alpha(n) \ll(\log n)^{-\lambda}$ (where $\lambda$ is as in the hypothesis of Theorem 2). Now Theorem 2 follows immediately from Lemmas 8 and 9 and Bradley [5, Theorem 4].

\section{Proof of Theorem 3}

Without loss of gencrality wc assumc that $\lambda_{n} \leqslant 1 \forall n \geqslant 1$, and that the sequence $\left\{a_{n}\right\}$ is strictly decreasing.

For each $n \geqslant 1$ define the positive number $\varepsilon_{n}:=\left(a_{n}-a_{n+1}\right) /(6 n)$.

Let $X^{(n)}:=\left\{X_{k}^{(n)}, k \in \mathbb{Z}\right\}, n=0,1,2, \ldots$, be strictly stationary sequences of r.v.'s with the following properties:

(3.1) These sequences $X^{(0)}, X^{(1)}, X^{(2)}, \ldots$ are independent of each other.

(3.2) The sequence $X^{(0)}$ satisfies $\alpha(n)=\lambda_{n} / 8$ and $\rho(n)=\lambda_{n} / 2$ for every $n \geqslant 1$. (This is possible by Theorem 6-and the two lines immediately following Theorem 6-in Bradley [4, page 4].) 
(3.3) For each $n=1,2,3, \ldots$ the sequences $X^{(n, j)}:=\left\{X_{k}^{(n)}, k \equiv j \bmod n\right\}, j=$ $1,2, \ldots, n$, are independent of each other.

(3.4) For each $n=1,2,3, \ldots$, each $j=1,2, \ldots, n$, the sequence $X^{(n, j)}$ defined in (3.3) is a strictly stationary Markov chain with state space $\{1,2,3,4\}$, with invariant marginal probability vector $\left[\left(1-\varepsilon_{n}\right)^{2},\left(1-\varepsilon_{n}\right) \varepsilon_{n},\left(1-\varepsilon_{n}\right) \varepsilon_{n}, \varepsilon_{n}^{2}\right]$, and with one-step transition probability matrix

$$
\left[\begin{array}{cccc}
1-\varepsilon_{n} & \varepsilon_{n} & 0 & 0 \\
0 & 0 & 1-\varepsilon_{n} & \varepsilon_{n} \\
1-\varepsilon_{n} & \varepsilon_{n} & 0 & 0 \\
0 & 0 & 1-\varepsilon_{n} & \varepsilon_{n}
\end{array}\right]
$$

(Thus the $(l, m)$-th entry of this matrix is $P\left(X_{(k+1) n+j}^{(n)}=m \mid X_{k n+j}^{(n)}=l\right)$.)

Note that for each $n \geqslant 1$, the distribution of the sequence $X^{(n)}$ is completely determined by (3.3) and (3.4). Also note that for each $n \geqslant 1$, each $j=1,2, \ldots, n$, the Markov chain $X^{(n, j)}$ is 1-dependent (one can see this by squaring the transition probability matrix). Hence for each $n \geqslant 1$ the sequence $X^{(n)}$ is $n$-dependent; this fact will be useful later on.

Let $f: \mathbb{R} \times \mathbb{R} \times \mathbb{R} \times \cdots \rightarrow \mathbb{R}$ be a bimeasurable isomorphism. (That is, both $f$ and $f^{-1}$ are Borel-measurable functions. Such an $f$ is well known to exist.) Define the sequence $X:=\left\{X_{k}, k \in \mathbb{Z}\right\}$ by

$$
X_{k}:=f\left(X_{k}^{(0)}, X_{k}^{(1)}, X_{k}^{(2)}, \ldots\right) \quad \forall k \in \mathbb{Z} .
$$

By an elementary argument, the sequence $X$ is strictly stationary. For $-\infty \leqslant J \leqslant$ $L \leqslant \infty$ define the $\sigma$-field

$$
\mathscr{F}_{J}^{L}:=\sigma\left(X_{k}, J \leqslant k \leqslant L\right)=\sigma\left(X_{k}^{(n)}, n \geqslant 0, J \leqslant k \leqslant L\right) .
$$

The last equality here holds by the properties of $f$.

We shall now prove that the sequence $X$ satisfies properties (i), (ii), (iii), and (iv) in Theorem 3. For arbitrary $\sigma$-fields $\mathscr{A}$ and $\mathscr{B}$ we shall use the notations

$$
\begin{aligned}
& \alpha(\mathscr{A}, \mathscr{B}):=\sup |P(A \cap B)-P(A) P(B)|, \quad A \in \mathscr{A}, B \in \mathscr{B} ; \\
& \rho(\mathscr{A}, \mathscr{B}):=\sup |\operatorname{Corr}(f, g)|, \quad f \in \mathscr{L}_{2}(\mathscr{A}), g \in \mathscr{L}_{2}(\mathscr{B}) .
\end{aligned}
$$

Proof of Theorem 3(i). For each $N=1,2,3, \ldots$ define the event $D_{N}$ by

$$
D_{N}:=\left\{X_{k}^{(n)}=1 \forall(n, k) \text { such that } n \geqslant N \text { and }-n+1 \leqslant k \leqslant 0\right\} .
$$

Now, for each $n=1,2,3, \ldots, P\left(X_{0}^{(n)}=1\right)=\left(1-\varepsilon_{n}\right)^{2} \geqslant 1-2 \varepsilon_{n}$. Hence, for each $n=$ $1,2,3, \ldots, P\left(X_{-n+1}^{(n)}=\cdots=X_{0}^{(n)}=1\right) \geqslant 1-2 n \varepsilon_{n}$. Hence, for each $N=1,2,3, \ldots$,

$$
P\left(D_{N}\right) \geqslant 1-\sum_{n=N}^{\infty} 2 n \varepsilon_{n}=1-2 \sum_{n=N}^{\infty}\left(a_{n}-a_{n+1}\right) / 6=1-a_{N} / 3 \geqslant \frac{1}{2} .
$$

(Recall that $a_{N} \leqslant a_{1} \leqslant \lambda_{1} \leqslant 1$.) By an elementary argument, for each $N=1,2,3, \ldots$ the probability measure $Q_{N}$ on $\mathscr{F}_{-\infty}^{\infty}$ defined by $Q_{N}(A):=P\left(A \mid D_{N}\right)$ has the following 
properties: Under $Q_{N}$ the sequences $X^{(0)}, X^{(1)}, X^{(2)}, \ldots$ are independent of each other, and the sequences $X^{(0)}, X^{(1)}, \ldots, X^{(N-1)}$ have the same distributions as they have under the given probability measure $P$. Thus under $Q_{N}$ the sequences $X^{(1)}, X^{(2)}, \ldots, X^{(N-1)}$ are each at most $(N-1)$-dependent. Also, under $Q_{N}$, for each $n \geqslant N$ the sequence $X^{(n)}$ has probability distribution given by $P\left(\cdot \mid X_{-n+1}^{(n)}=\right.$ $\cdots=X_{0}^{(n)}=1$ ) and in particular (under $\left.Q_{N}, \forall n \geqslant N\right) \sigma\left(X_{k}^{(n)}, k \leqslant 0\right)$ and $\sigma\left(X_{k}^{(n)}, k \geqslant\right.$ 1) are independent $\sigma$-fields. Thus under $Q_{N}$ the $\sigma$-fields $\sigma\left(X^{(0)}\right), \sigma\left(X_{k}^{(n)}, n \geqslant 1\right.$, $k \leqslant 0)$, and $\sigma\left(X_{k}^{(n)}, n \geqslant 1, k \geqslant N\right)$ are independent. Consequently, using (3.2), $\forall N \geqslant 1$

$$
\begin{aligned}
\rho_{Q_{N}}\left(\mathscr{F}_{-\infty}^{0}, \mathscr{F}_{N}^{\infty}\right) & =\rho_{Q_{N}}\left(\sigma\left(X_{k}^{(0)}, k \leqslant 0\right), \sigma\left(X_{k}^{(0)}, k \geqslant N\right)\right) \\
& =\rho_{P}\left(\sigma\left(X_{k}^{(0)}, k \leqslant 0\right), \sigma\left(X_{k}^{(0)}, k \geqslant N\right)\right)=\lambda_{N} / 2 .
\end{aligned}
$$

The first equality here follows from the well known fact that if $\mathscr{A}_{1} \mathscr{B}_{1}, \mathscr{A}_{2}$, and $\mathscr{B}_{2}$ are $\sigma$-fields on some probability space and $\mathscr{A}_{1} \vee \mathscr{B}_{1}, \mathscr{A}_{2}$, and $\mathscr{B}_{2}$ are independent, then $\rho\left(\mathscr{A}_{1} \vee \mathscr{A}_{2}, \mathscr{B}_{1} \vee \mathscr{B}_{2}\right)=\rho\left(\mathscr{A}_{1}, \mathscr{B}_{1}\right)$. This fact is a simple consequence of the identity $\rho(\mathscr{A}, \mathscr{B})=\sup \|E(f \mid \mathscr{B})-E f\|_{2} /\|f\|_{2}, f \in \mathscr{L}_{2}(\mathscr{A})$. Thus $\forall N \geqslant 1$ one has that

$$
\begin{aligned}
\forall A \in \mathscr{F}_{-\infty}^{0} \forall B \in \mathscr{F}_{N}^{\infty} \\
\quad\left|Q_{N}(A \cap B)-Q_{N}(A) Q_{N}(B)\right| \leqslant\left(\lambda_{N} / 2\right)\left[Q_{N}(A) Q_{N}(B)\right]^{1 / 2} .
\end{aligned}
$$

By (3.6) and an elementary argument, $\forall N \geqslant 1 \forall A \in \mathscr{F}_{-\infty}^{\infty}$, one has that

$$
\left|Q_{N}(A)-P(A)\right|=\left|P\left(A \mid D_{N}\right)-P(A)\right| \leqslant 1-P\left(D_{N}\right) \leqslant a_{N} / 3 .
$$

Hence by (3.7) and (3.6), one has that $\forall N=1,2,3, \ldots \forall A \in \mathscr{F}_{-\infty}^{0} \forall B \in \mathscr{F}_{N}^{\infty}$,

$$
\begin{aligned}
&|P(A \cap B)-P(A) P(B)| \\
& \leqslant\left|P(A \cap B)-Q_{N}(A \cap B)\right|+\left|Q_{N}(A \cap B)-Q_{N}(A) Q_{N}(B)\right| \\
&+\left|Q_{N}(A)\left[Q_{N}(B)-P(B)\right]\right|+\left|P(B)\left[Q_{N}(A)-P(A)\right]\right| \\
& \leqslant a_{N}+\left|Q_{N}(A \cap B)-Q_{N}(A) Q_{N}(B)\right| \\
& \leqslant a_{N}+\left(\lambda_{N} / 2\right)\left[Q_{N}(A) Q_{N}(B)\right]^{1 / 2} \\
& \leqslant a_{N}+\left(\lambda_{N} / 2\right)\left[\frac{P(A)}{P\left(D_{N}\right)} \cdot \frac{P(B)}{P\left(D_{N}\right)}\right]^{1 / 2} \\
& \leqslant a_{N}+\lambda_{N}[P(A) P(B)]^{1 / 2} .
\end{aligned}
$$

This completes the proof of Theorem 3(i).

Proof of Theorem 3(ii). For each $n \geqslant 1$,

$$
\begin{aligned}
\lambda_{n} / 8 & =\alpha\left(\sigma\left(X_{k}^{(0)}, k \leqslant 0\right), \sigma\left(X_{k}^{(0)}, k \geqslant n\right)\right) \\
& \leqslant \alpha\left(\mathscr{F}_{-\infty}^{0}, \mathscr{F}_{n}^{\infty}\right) \leqslant a_{n}+\lambda_{n} \leqslant 2 \lambda_{n}
\end{aligned}
$$

by (3.2), Theorem 3(i), and the assumption $a_{n} \leqslant \lambda_{n}$. Thus (ii) holds. 
Proof of Theorem 3(iii). For each $n \geqslant 1$,

$$
\operatorname{Corr}\left(I\left(X_{0}^{(n)}=2 \text { or } 4\right), I\left(X_{n}^{(n)}=3 \text { or } 4\right)\right)=1
$$

by an elementary calculation, and hence $\rho\left(\mathscr{F}_{-\infty}^{0}, \mathscr{F}_{n}^{\infty}\right)=1$.

Proof of Theorem 3(iv). Recall the elementary fact that if (1.2) holds then $\alpha(n) \leqslant$ $a_{n}+\lambda_{n}, n=1,2, \ldots$. By essentially the same elementary argument, if hypothesis (a) in (iv) holds then the conclusion of (iv) follows quickly from Theorem 3(ii). Now let us assume that hypothesis (b) in (iv) holds, namely $a_{n}^{*}=o\left(a_{n}\right)$ and $\lambda_{n}^{*} \rightarrow 0$; we shall show that under these assumptions too the conclusion of (iv) holds.

For each $n \geqslant 1$ define the events $A_{n}$ and $B_{n}$ by

$$
\begin{aligned}
& A_{n}:=\left\{X_{k}^{(n)}=2 \text { or } 4 \text { for some integer } k,-n / 2<k \leqslant 0\right\}, \\
& B_{n}:=\left\{X_{k}^{(n)}=3 \text { or } 4 \text { for some integer } k, n / 2<k \leqslant n\right\} .
\end{aligned}
$$

By an elementary calculation, $\forall n \geqslant 1 P\left(A_{n}\right)=P\left(B_{n}\right)$ and $P\left(B_{n} \mid A_{n}\right)=1$; and also $P\left(A_{n}\right) \sim n \varepsilon_{n} / 2$ as $n \rightarrow \infty$. (Here and in what follows, the notation $c_{n} \sim d_{n}$ means Lim $c_{n} / d_{n}=1$.)

For each $N=1,2,3, \ldots$ define the events $A_{N}^{*}$ and $B_{N}^{*}$ by

$$
\begin{aligned}
& A_{N}^{*}:=A_{N} \cup A_{N+1} \cup A_{N+2} \cup \cdots, \\
& B_{N}^{*}:=B_{N} \cup B_{N+1} \cup B_{N+2} \cup \cdots,
\end{aligned}
$$

By an elementary calculation, $\forall N \geqslant 1 P\left(A_{N}^{*}\right)=P\left(B_{N}^{*}\right)$ and $P\left(B_{N}^{*} \mid A_{N}^{*}\right)=1$; and also, as $N \rightarrow \infty, P\left(A_{N}^{*}\right) \sim \sum_{n=N}^{\infty} n \varepsilon_{n} / 2=a_{N} / 12$.

Hence, for each $n=1,2,3, \ldots, A_{2 n}^{*} \in \mathscr{F}_{-\infty}^{0}$ and $B_{2 n}^{*} \in \mathscr{F}_{n}^{\infty}$; and as $n \rightarrow \infty$,

$$
\begin{aligned}
\left|P\left(A_{2 n}^{*} \cap B_{2 n}^{*}\right)-P\left(A_{2 n}^{*}\right) P\left(B_{2 n}^{*}\right)\right| & =P\left(A_{2 n}^{*}\right)\left[1-P\left(A_{2 n}^{*}\right)\right] \sim P\left(A_{2 n}^{*}\right) \\
& =\left[P\left(A_{2 n}^{*}\right) P\left(B_{2 n}^{*}\right)\right]^{1 / 2} \sim a_{2 n} / 12 .
\end{aligned}
$$

Since $a_{n} \ll a_{2 n}$ by the hypothesis of Theorem 3, it is now easy to see that if hypothesis (b) in Theorem 3(iv) holds, then the conclusion of (iv) also holds. This completes the proof of (iv).

\section{Acknowledgment}

The authors thank E. Presutti for his very useful suggestions, which stimulated this research.

\section{References}

[1] H.C.P. Berbee, Random walks with stationary increments and renewal theory (Mathematical Centre, Amsterdam, 1979). 
[2] P. Billingsley, Convergence of Probability Measures (Wiley, New York, 1968).

[3] C. Boldrighini, A. De Massi, A. Nogueira and E. Presutti, The dynamics of a particle interacting with a semi-infinite ideal gas in a Bernoulli flow, in: Statistical Physics and Dynamical Systems (Progress in Physics) (Birkhäuser, 1985) p. 153.

[4] R.C. Bradley, Central limit theorems under weak dependence, J. Multivar. Anal. 11 (1981) 1-16.

[5] R.C. Bradley, Approximation theorems for strongly mixing random variables, Michigan Math. J. 30 (1983) 69-81.

[6] R.C. Bradley, Equivalent measures of dependence, J. Multivar. Anal. 13 (1983) 167-176.

[7] R.C. Bradley and W. Bryc, Multilinear forms and measures of dependence between random variables, J. Multivar. Anal. 16 (1985) 335-367.

[8] A.V. Bulinskii, On measures of dependence close to the maximal correlation coefficient, Soviet Math. Dokl. 30 (1984) 249-252.

[9] Y.A. Davydov, Convergence of distributions generated by stationary stochastic processes, Theory Probab. Appl. 13 (1968) 691-696.

[10] Y.A. Davydov, The invariance principle for stationary processes, Theory Probab. Appl. 15 (1970) 487-498.

[11] H. Dehling and W. Philipp, Almost sure invariance principles for weakly dependent vector-valued random variables, Ann. Probab. 10 (1982) 689-701.

[12] M. Denker and W. Philipp, Approximation by Brownian motion for Gibbs measures and flows under a function, Ergodic Theory Dynamical Systems 4 (1984) 541-552.

[13] C.H. Deo, A note on empirical processes of strong mixing sequences, Ann. Probab. 1 (1973) 870-875.

[14] J.L. Gastwirth and H. Rubin, The asymptotic distribution theory of the empiric c.d.f. for mixing stochastic processes, Ann. Statist. 3 (1975) 809-824.

[15] N. Herrndorf, A functional central limit theorem for weakly dependent sequences of random variables, Ann. Probab. 12 (1984) 141-153.

[16] N. Herrndorf, A functional central limit theorem for $\rho$-mixing sequences, J. Multivar. Anal. 15 (1984) 141-146.

[17] I.A. Ibragimov, A note on the central limit theorem for dependent random variables, Theory Probab. Appl. 20 (1975) 135-141.

[18] I.A. Ibragimov and Y.V. Linnik, Independent and Stationary Sequences of Random Variables (Wolters-Noordhoff, Groningen, 1971).

[19] E. Lehmann, Some concepts of dependence, Ann. Math. Statist. 37 (1966) 1137-1153.

[20] H. Oodaira and K. Yoshihara, Functional central limit theorems for strictly stationary processes satisfying the strong mixing condition, Kôdai Math. Sem. Rep. 24 (1972) 259-269.

[21] M. Peligrad, Invariance principles for mixing sequences of random variables, Ann. Probab. 10 (1982) 968-981.

[22] M. Peligrad, Invariance principles under weak dependence, J. Multivar. Anal. (to appear).

[23] W. Philipp and W. Stout, Almost sure invariance principles for partial sums of weakly dependent random variables, Mem. Amer. Math. Soc. No. 161 (1975).

[24] P. Shields, The Theory of Bernoulli Shifts (University of Chicago Press, Chicago, 1973).

[25] V.A. Volkonskii and Y.A. Rozanov, Some limit theorems for random functions I, Theory Probab. Appl. 4 (1959) 178-197.

[26] K. Yoshihara, Probability inequalities for sums of absolutely regular processes and their applications, Z. Wahrsch. Verw. Gebiete 43 (1978) 319-329. 